\title{
DIFICULDADES DE ESTIMATIVA DOS PARÂMETROS DE RUGOSIDADE AERODINÂMICA PELO PERFIL LOGARÍTMICO DO VENTO SOBRE VEGETAÇÃO ESPARSA EM REGIÃO SEMI-ÁRIDA
}

\author{
Gustavo Bastos Lyra ${ }^{1}$ e Antonio Roberto Pereira² \\ Recebido em 2 abril, 2007 / Aceito em 5 junho, 2007 \\ Received on April 2, 2007 / Accepted on June 5, 2007
}

\begin{abstract}
The zero-plane displacement $(d)$ and the roughness length $\left(z_{0}\right)$ of sparse shrubs in semi-arid region were determined by the conventional (statistical) method during the HAPEX-Sahel experiment. Measurements of the wind profile at four levels above of surface (3.0, 4.1, 5.3 and $8.5 \mathrm{~m})$ and eddy correlation turbulent fluxes determined at $9 \mathrm{~m}$ were used. The method was applied in neutral atmospheric conditions, with stability parameter $\zeta<|0.0325|$, wind speed $>1 \mathrm{~m} \mathrm{~s}^{-1}$ in the lower level and the friction velocity $>0.1 \mathrm{~m} \mathrm{~s}^{-1}$ determined by eddy correlations. Approximately $4.3 \%$ of the observation satisfied these restrictions, but only $1 \%$ had adequate fetch conditions. The value of $d$ was equal to $1.09 \pm 0.14 \mathrm{~m}$ and it represented about $53 \%$ of the height of the vegetation $(h)$ and $z_{0}=0.184 \pm 0.017 \mathrm{~m}=$ $0.089 \mathrm{~h}$. The data selection criteria used was satisfactory, but the applicability of the method is restricted by the difficulties in observing adiabatic conditions and adequate fetch. Alternative estimates with a single height of measurement above the inertial sublayer gave physically inconsistent estimates of $d$ and $z_{0}$.
\end{abstract}

Keywords: zero-plane displacement, roughness length, roughness sublayer, fetch, HAPEX-Sahel.

RESUMO. 0 deslocamento do plano zero $(d)$ e o comprimento de rugosidade $\left(z_{0}\right)$ de arbustos esparsos em região semi-árida foram determinados pelo método convencional (ajuste estatístico), com medidas realizadas durante 0 experimento HAPEX-Sahel. Utilizaram-se medidas do perfil de vento obtidas em quatro níveis acima da superfície (3,0; 4,1; 5,3 e 8,5 m) e dos fluxos determinados por correlação de vórtices (9,0 m). 0 método foi aplicado a observações próximas da neutralidade atmostérica, com parâmetro $\zeta$ de estabilidade $<|0,0325|$, juntamente com a velocidade do vento $>1 \mathrm{~m} \mathrm{~s}^{-1}$ no nível inferior de medida e a de fricção $>0,1 \mathrm{~m} \mathrm{~s}^{-1}$. Aproximadamente 4,3\% das observações satisfizeram essas restrições, mas apenas $1 \%$ teve bordadura (fetch) adequada. 0 valor de $d$ estimado foi igual a 1,09 \pm $0,14 \mathrm{~m}$ e representou $53 \%$ da altura da vegetação $(h)$ e $z_{0}=0,184 \pm 0,017 \mathrm{~m}=0,089 h$. Os critérios de seleção de dados foram adequados ao tipo de vegetação, mas a aplicabilidade do método é restrita pela dificuldade em se observar condições próximas à neutralidade atmosférica e bordadura adequada. Estimativas baseadas em apenas um nível de medida acima da subcamada inercial resultaram em valores fisicamente inconsistentes de $d$ e $z_{0}$.

Palavras-chave: deslocamento do plano zero, comprimento de rugosidade, subcamada rugosa, bordadura, HAPEX-Sahel.

\footnotetext{
${ }^{1}$ Universidade Federal de Alagoas, Instituto de Ciências Atmostéricas, Cidade Universitária, Km 14 Rodovia BR-104, Tabuleiro dos Martins - 57072-970 Maceió, AL, Brasil. Tel.: (82) 3214-1360 - E-mail: gblyra@gmail.com

2Universidade de São Paulo, Escola Superior de Agricultura "Luiz de Queiroz", Departamento de Ciências Exatas, Av. Pádua Dias, 11, Caixa Postal 9 - 13418-900 Piracicaba, SP, Brasil. Tel.: (19) 3429-4123 - E-mail: arperei@@esalq.usp.br
} 


\section{INTRODUÇÃO}

A definição da rugosidade aerodinâmica de uma superfície é fundamental em escala micrometeorológica e em parametrizações de modelos globais e de mesoescala (de Bruin \& Verhoef, 1997; Takagi et al., 2003; Zhang et al., 2004, Tsai \& Tsuang, 2005). A rugosidade aerodinâmica pode ser representada pelo comprimento de rugosidade $\left(z_{0}\right)$ e pelo deslocamento do plano zero $(d)$, incorporados ao perfil logarítmico do vento acima da superfície, i.e.,

$$
U_{z}=\left(\frac{u *}{k}\right) \ln \left(\frac{z-d}{z_{0}}\right) \quad z>z *
$$

em que $z$ é altura de medida da velocidade horizontal do vento $\left(U_{z}\right) ; u_{*}$ é a velocidade de fricção; $k$ é a constante de von Kármán e $z_{*}$ é o limite superior da subcamada atmosférica rugosa (SCR).

Freqüentemente, $z_{0}$ e $d$ são estimados pelo método gráfico ou estatístico (método convencional), com ajuste iterativo da eq. 1 em condições adiabáticas (Robinson, 1962; Stearns, 1970). Pela lei logarítmica do vento, $z_{0}$ e $d$ são inter-relacionadas, não resultando necessariamente em solução física adequada (Jacobs \& van Boxel, 1988; Schaudt, 1998; Tsai \& Tsuang, 2005).

Nesses métodos é necessário que observações acuradas de $U_{z}$ se restrinjam à subcamada inercial da camada limite de fluxo constante ou de equilíbrio para satisfazer as condições de validade do perfil logarítmico (Monteith \& Unsworth, 1990; Wieringa, 1993; de Bruin \& Verhoef, 1997; Britter \& Hanna, 2003; Tsai \& Tsuang, 2005; Verkaik \& Holtslag, 2007). Ou seja, o nível inferior de medida deve ser acima da subcamada rugosa $\left(z>z_{*}\right.$ ) e a bordadura (fetch) com extensão entre 50 e 100 vezes a maior altura de medida de $U_{z}$ (Wieringa, 1993; de Bruin \& Verhoef, 1997). Quando não satisfeitas essas restrições, ou quando aplicado em condições diabáticas sem as correções do perfil de vento, sobre baixas velocidades do vento ou quando poucos níveis de medida são utilizados, o método convencional produz resultados incoerentes (Raupach et al., 1980; Molion \& Moore, 1983; Wieringa, 1993).

Acima da subcamada rugosa, o fluxo não é influenciado significativamente pela viscosidade ou pela estrutura individual dos obstáculos. 0 fluxo na $S C R$ varia em três dimensões, em função da proximidade dos elementos rugosos, com transferência vertical de momentum denominada de "dissipativa", resultado do movimento não turbulento, dependente da localização individual dos elementos rugosos (Raupach \& Thom, 1981; Tsai \& Tsuang, 2005). Enquanto na camada limite de fluxo constante, os fluxos estão em equilíbrio dinâmico, com desvio padrão normalizado do vento $\left(\sigma_{u} / u_{*}\right)$ e coeficiente de arrasto normalizado independente da altura e coeficiente adimensional de transporte turbulento para momentum $\left(\phi_{m}\right)$ igual a unidade, em condições adiabáticas, ou seja, a teoria de similaridade de Monin-Obukhov é válida (Verkaik \& Holtslag, 2007). A espessura da camada limite de equilíbrio depende das condições de estabilidade e da rugosidade aerodinâmica da superfície (Wieringa, 1993; Tsai \& Tsuang, 2005).

Acima de obstáculos esparsos e de porte alto, como no caso de arbustos, florestas ou áreas urbanas, as condições de validade do perfil são difíceis de serem observadas. As dificuldades se devem principalmente à formação da $S C R$ próximo ao topo dos obstáculos, ao efeito de proteção aerodinâmica entre os elementos rugosos ou à bordadura inadequada (Molion \& Moore, 1983; de Bruin \& Moore, 1985; Lloyd et al., 1992; de Bruin \& Verhoef, 1997; Tsai \& Tsuang, 2005).

A região do Sahel forma a borda sul do deserto do Saara, África, apresentando características semi-áridas de savana, sendo importante fonte de calor sensível para a atmosfera. A região é escassamente vegetada, com gramíneas anuais e arbustos perenes aleatoriamente distribuídos ao norte, que gradualmente se misturam às savanas com gramíneas perenes e árvores dispersas, e a extensas áreas agrícolas ao sul. Suas características de vegetação esparsa e heterogênea, associadas ao elevado fluxo de calor sensível, típico de regiões semi-áridas, e a baixas velocidades do vento (Morgan et al., 1971; de Bruin \& Verhoef, 1997), dificultam a observação de condições próximas à atmosfera neutra, o que restringe a utilização do método convencional. Assim estudos na região permitem compreender a dinâmica do transporte turbulento em regiões semi-áridas e com vegetação esparsa e heterogênea.

0 objetivo do presente trabalho é avaliar as dificuldades de estimativa do deslocamento do plano zero e do comprimento de rugosidade pelo método convencional (ajuste estatístico) para vegetação de porte alta e esparsa em região semi-árida.

\section{MATERIAL E MÉTODOS Área experimental}

Medidas micrometeorológicas e da vegetação foram feitas no subsítio Fallow Bush do Super-Sítio Sul (SSS) do HAPEX-Sahel (Hydrological and Atmospheric Pilot Experiment in the Sahe/). 0 HAPEX-Sahel foi conduzido numa região semi-árida de savana no Niger, oeste da África. A área de $S S S(0,8 \times 1,0 \mathrm{~km})$ era limitada ao norte, nordeste e noroeste por áreas de floresta nativa (Tiger Bush) com altura da vegetação entre 4 e $6 \mathrm{~m}$ e, nas outras direções, por campos de milheto com altura média de 2,6 m (Wallace et al., 1994). A vegetação de SSS era escassa e divi- 
dida em duas camadas: uma formada por gramíneas e herbáceas; outra composta por arbustos lenhosos, com distribuição espacial esparsa e predominância de Guiera senegalensis L., mas com algumas Combretum micranthum G. Don (Levy \& Jarvis, 1999).

Avaliações da estrutura física de 220 arbustos ( $n$ ) de Guiera senegalensis L. foram feitas em fevereiro de 1992, em uma área de $6.750 \mathrm{~m}^{2}\left(A_{T}\right)$. 0 ponto central da área de análise da estrutura dos arbustos estava a $120 \mathrm{~m}$ do mastro micrometeorológico. Determinou-se a altura máxima ( $h$, em $\mathrm{m}$ ), a altura do raio máximo $\left(h_{\max }\right.$, em m) e o raio máximo ( $r_{\max }$, em $\left.\mathrm{m}\right)$ da copa. Avaliações mensais semidiretas do índice de área foliar (IAF; área total de um único lado de todas as folhas pela área de superfície do solo disponível à planta) e do índice de área superficial dos galhos ( $/ A G$; área de superfície de todos os galhos por área de superfície do solo disponível à planta) foram feitas entre junho e outubro de 1992. Havia cerca de 327 arbustos/ha de Guiera senegalensis L. com $h=2,06 \pm 0,47 \mathrm{~m}, r_{\max }=1,57 \pm$ $0,56 \mathrm{~m}, I A F=0,365 \mathrm{~m}^{2} \mathrm{~m}^{-2}$ e $I A G=0,125 \mathrm{~m}^{2} \mathrm{~m}^{-2}$. Detalhes das análises da estrutura física da vegetação são apresentados por Levy \& Jarvis (1999).

\section{Medidas micrometeorológicas}

Medidas micrometeorológicas foram obtidas entre 20/08 e 30/09/92, no período de transição da estação úmida para a seca (Wallace et al., 1994). 0 perfil de velocidade horizontal do vento foi avaliado por quatro anemômetros de canecas (A100H, VECTOR INSTRUMENTS, North Wales, UK) dispostos a 3,0; 4,1; 5,3 e 8,5 $\mathrm{m}$ acima do solo. Para superfície moderadamente rugosa $\left(z_{0} \approx 0,1 \mathrm{~m}\right)$ esse número de níveis de medidas é considerado adequado para a determinação de $z_{0} \mathrm{e} d$ pelos métodos baseados na lei logarítmica do vento (Kustas et al., 1989; Wieringa, 1993). 0 saldo de radiação $\left(R_{n}\right)$ foi medido por dois saldos radiômetros (CN1-R, MIDDLETON SOLAR, Victoria, AU), sendo um sobre os arbustos e outro acima das gramíneas/herbáceas, ambos a $3 \mathrm{~m}$ acima do solo, obtendo-se um valor médio. 0 fluxo de calor no solo $(G)$ foi medido por oito placas de fluxo de calor em pares (CN3, MIDDLETON SOLAR, Victoria, AU), a 0,05 m de profundidade de cada tipo de vegetação, obtendo-se um valor médio da área. As medidas foram feitas em intervalos de 30 s e suas médias armazenadas a cada 10 min em datalogger (CR21X, CAMPBELL SCIENTIFIC Inc., Logan, UT).

Os componentes vertical $(w)$, longitudinal $(u)$ e meridional $(v)$ da velocidade do vento foram obtidos por um anemômetro sônico 3D (GILL R3, SOLENT, Southampton, UK). A temperatura do ar $(T)$ foi medida por um termômetro resistivo de platina (Department of Meteorology, UNIVERSITY OF READING, Reading, UK) e um higrômetro a infravermelho (IR-2000, OPHIR CORPORATION, Lakewood, CO) media a umidade específica do ar $(q)$. Esses instrumentos estavam no topo de um mastro e a $9 \mathrm{~m}$ acima do solo. As covariâncias médias entre as flutuações das variáveis supracitadas foram calculadas a cada 10 min de medidas em freqüência de $20 \mathrm{~Hz}$ e armazenadas em datalogger (Wallace et al., 1994; Lloyd et al., 1997). Correções para a rotação das coordenadas, freqüência de resposta limitada dos sensores, média do comprimento do trajeto, separação lateral e longitudinal do sensor e freqüência de resposta do sistema de aquisição de dados foram feitas conforme Lloyd et al. (1997).

\section{Estimativas dos parâmetros de rugosidade aerodinâmica da superfície}

Na estimativa de $d$ e $z_{0}$ utilizaram-se médias de 30 min de todos os elementos micrometeorológicos, como recomendado por Monteith \& Unsworth (1990). Por regressão linear simples de $U_{z}$ em função de $\ln (z-d)$, variou-se $d$ até que o coeficiente de determinação $\left(r^{2}\right)$ fosse o maior possível. Posteriormente, determinou-se a velocidade de fricção $\left(u_{*}\right)$ e $z_{0}$ pelos valores do intercepto ( $a$ ) e da inclinação $(b)$ dessa regressão, pelas seguintes relações analíticas:

$$
\begin{aligned}
& u_{*}=k b \\
& z_{0}=\exp \left(-\frac{a}{b}\right) .
\end{aligned}
$$

Utilizou-se $k=0,40$ (Wieringa, 1993) e considerou-se o valor inicial das iterações como $d=0,70 h$.

\section{Condições de neutralidade atmosférica}

As observações utilizadas nas análises foram restritas a condições próximas da neutralidade atmosférica para satisfazer a lei do perfil logarítmico do vento. Selecionaram-se observações que apresentaram módulo da energia disponível $\left|R_{n}-G\right|<20 \mathrm{~W} \mathrm{~m}^{-2} \mathrm{e}$ do fluxo de calor sensível determinado por correlação de vórtices $H<15 \mathrm{~W} \mathrm{~m}^{-2}$, similar a de Bruin \& Moore (1985). Devido a erros proporcionados pela inércia dos anemômetros de canecas, perfis com velocidade do vento no anemômetro inferior $(z=$ $3,0 \mathrm{~m}$ ) menor que $1,0 \mathrm{~m} \mathrm{~s}^{-1}$ e $u_{*}$ determinada por correlação de vórtices $<0,1 \mathrm{~m} \mathrm{~s}^{-1}$ também foram excluídos, seguindo os procedimentos de Shuttleworth et al. (1988).

Satisfeitas as condições acima, avaliou-se a estabilidade atmosférica no nível de medida do anemômetro sônico (9,0 m) pelo parâmetro $\zeta$ de estabilidade (Monin \& Obukhov, 1954). Utilizaram-se apenas observações que apresentaram $|\zeta|<$ 
0,0325. 0 parâmetro $\zeta$ foi estimado pela seguinte relação:

$$
\zeta=\frac{z}{L}=\frac{z k g \overline{w^{\prime} \theta^{\prime}}}{u_{*}^{3} \theta}
$$

em que $L$ (em $\mathrm{m}$ ) é a escala de comprimento (altura) de MoninObukhov; $g\left(=9,81 \mathrm{~m} \mathrm{~s}^{-2}\right)$ é a aceleração devido a gravidade; $\theta$ (em K) é a temperatura potencial e $\overline{w^{\prime} \theta^{\prime}}\left(\mathrm{em} \mathrm{m} \mathrm{s}^{-1} \mathrm{~K}\right)$ é a covariância média entre as flutuações da componente vertical do vento e da temperatura potencial do ar, representando o fluxo de calor sensivel da superfície.

\section{Subcamada inercial}

Avaliou-se o nível inferior de medida de $U_{z}(3,0 \mathrm{~m})$ em relação à base da subcamada inercial e se a bordadura ( fetch) foi adequada para o nível superior ( $8,5 \mathrm{~m}$ ) de medida, ou seja, se esses níveis se encontravam no interior da subcamada inercial.

Considerou-se a base da SCl como o topo da subcamada rugosa (Monteith \& Unsworth, 1990), sendo esse determinado pelos modelos abaixo:

$$
\begin{array}{ll}
z_{*}-d=c_{w}(h-d) & \text { (Raupach, 1992) } \\
z_{*}=1,5 h & \text { (Wieringa, 1993) } \\
z_{*}=d+10 z_{0} & \text { (Jacobs \& van Boxel, 1988) }
\end{array}
$$

em que $z_{*}(\mathrm{~m})$ é 0 topo da subcamada rugosa e $c_{w}=2$ (Raupach, 1992).

A bordadura necessária para que o nível superior de medida do perfil de vento esteja no interior da $S C /$, completamente adaptada à rugosidade da superfície, foi determinada pelo modelo de Miyake modificado por Wieringa (1993). Esse modelo considera que os fluxos estão próximos ao equilíbrio a 0,1 da camada limite atmosférica interna, pela seguinte relação:

$$
X=2 z_{0}\left[\frac{10 z}{z_{0}}\left(\ln \frac{10 z}{z_{0}}-1\right)+1\right]
$$

em que $X(\mathrm{~m})$ éa distância da bordadura na direção predominante do vento. Considerou-se $z(=8,5 \mathrm{~m})$ como a altura superior de medida de $U_{z}$.

\section{Análise estatística}

Utilizaram-se nas análises dos valores estimados de $d$ e $z_{0}$ a análise de regressão linear entre $u_{*}$ determinado por correlação de vórtices e estimado pela lei logarítmica do vento. A velocidade de fricção foi estimada para cada nível de medida $(3,0 ; 4,1 ; 5,3$ e $8,5 \mathrm{~m}$ ) em função dos parâmetros de rugosidade e de $U_{z}$. Como na $S C l u_{*}$ é independe da altura, utilizou-se nas análises a média das estimativas de $u_{*}$ para os quatro níveis de medida de $U_{z}$ a cada observação.

Determinou-se também a raiz do quadrado médio do erro ( $R Q M E, \mathrm{em} \mathrm{m} \mathrm{s}^{-1}$ ) e a concordância entre os valores de $u_{*}$ observados e estimados pelo perfil logarítmico, utilizando o índice de concordância de Willmott ( $I$ ) (Willmott, 1981). Efetuaram-se os testes $F$ e $t$ para, respectivamente, a variância e a média de $u_{*}$ estimada em função de $d$ e $z_{0}$ obtidos pelo método convencional e para os valores determinados por correlação de vórtices.

\section{RESULTADOS E DISCUSSÃO}

Foram obtidas 600 observações médias de 30 min com medidas simultâneas do perfil da velocidade horizontal do vento e correlação de vórtices turbulentos, mas apenas 26 delas $(\sim 4,3 \%)$ tinham condições próximas à neutralidade atmostérica $(|\zeta|<0,0325)$. Em regiões semi-áridas são difíceis de serem observadas condições de neutralidade, em função do elevado fluxo de calor sensível, com forte turbulência originada por flutuação térmica (Morgan et al., 1971; de Bruin \& Verhoef, 1997). Na região do Sahel, associado aos elevados valores de $H$, observam-se baixas velocidades do vento (de Bruin \& Verhoef, 1997), o que inibi a geração da energia mecânica turbulenta, e dessa forma, maior dificuldade em se registrar condições adiabáticas na região.

Das observações próximas à adiabática, 20 (76,9\%) foram nos horários próximos ao nascer do sol (de 05:00 às 07:00 HL, horário local) e ao ocaso (17:00 e 19:30 HL), sendo a maior parte delas (11) entre 17:00 e 19:30 h. Próximo ao nascer e ao ocaso, condições de atmosfera neutra são observadas com maior freqüência devido à inversão no sentido do fluxo de calor sensível, com baixos valores de $H$. Entretanto, próximo ao nascer do sol notam-se elevadas taxas de variação de $H$ com 0 tempo, função do intenso aquecimento solar, enquanto nos horários adjacentes ao ocaso essa variação é gradual (Morgan et al., 1971), o que justifica a maior proporção de condições adiabáticas próximas ao ocaso em relação ao nascer do sol.

Os fatores de correção diabático do perfil de vento (Yasuda, 1988) aplicados aos extremos observados de $\zeta(-0,0323 \mathrm{e}$ $0,0031)$ resultaram em desvios da lei logarítmica do vento proporcionais a $-3,09 \%$ (instabilidade) e $0,37 \%$ (estabilidade) de $U_{z}$ medida pelo anemômetro sônico. Esses valores são próximos da acurácia dos anemômetros de caneca utilizados ( $\pm 1 \%$ ) e inferiores ao citado por Wieringa (1980) para a superestimativa desse tipo de anemômetro ( 5\%). Em termos de valor absoluto, os desvios extremos devido à estabilidade foram de $-0,10 \mathrm{e}$ 
$0,02 \mathrm{~m} \mathrm{~s}^{-1}$, que em módulo mostraram-se inferiores a inércia dos anemômetros $\left(0,2 \mathrm{~m} \mathrm{~s}^{-1}\right)$. Intervalo similar de correções e da ordem de $\pm 0,05 \mathrm{~m} \mathrm{~s}^{-1}$ foram determinado por Kustas et al. (1989), e assumido como desprezível para estimativa dos parâmetros de rugosidade pela lei logarítmica. Deste modo, a faixa de neutralidade observada foi considerada adequada para determinação dos parâmetros de rugosidade pelo método convencional, sem a necessidade de aplicação das correções diabáticas ao perfil.

A utilização do método convencional $(\mathrm{CO})$ resultou em $d$ negativo em 17 observações e três mostraram $z_{0} / d>1$, sendo inconsistentes fisicamente e desconsiderados das análises. Pelas observações consideradas ( $1 \%$ dos dados válidos), obteve-se $d=1,09 \pm 0,14 \mathrm{~m} \mathrm{e} z_{0}=0,184 \pm 0,017 \mathrm{~m}$. 0 coeficiente de variação $(c v)$ foi de $13 \%$ para $d$ e $9,5 \%$ para $z_{0}$, sendo similares aos valores observados por Munro \& Oke (1973) para a cultura do trigo. Contudo, inferiores aos determinados por Dolman (1986) para floresta de carvalho (100\% para $z_{0}$ e $30 \%$ para $d$ ) e aos observados por Kustas et al. (1989) num dossel esparso de algodão e em torno de $65 \%$ para ambos, $d$ e $z_{0}$.

A relação entre $U_{z} \mathrm{e} \ln (z-d)$ apresentou ajuste estatístico satisfatório do perfil para $d$ calculado a cada observação considerada, com coeficiente de determinação superior a 0,99, indicado por Takagi et al. (2003) como limite mínimo aceitável.

Os valores de $d$ negativo ou inferior a $z_{0}$ resultaram em valores elevados de $z_{0}(>0,480 \mathrm{~m})$, devida à correlação inversamente proporcional desses parâmetros quando determinados pela lei logarítmica (Jacobs \& van Boxel, 1988; Schaudt, 1998; Tsai \& Tsuang, 2005). Os valores inconsistentes de $d$ e $z_{0}$ podem ter sido proporcionados pela influência da estabilidade na forma do perfil, por baixas velocidades do vento ou por medidas fora da $S C l$ (Molion \& Moore, 1983; Wieringa, 1993; de Bruin \& Verhoef, 1997; Britter \& Hanna, 2003; Tsai \& Tsuang, 2005; Verkaik \& Holtslag, 2007).

A estabilidade atmosférica influencia na forma do perfil do vento, divergindo da lei logarítmica. Teoricamente, a tendência é de decréscimo da turbulência em condições de estabilidade, produzindo gradientes verticais de $U_{z}$ com inclinação superior aos observados sobre atmosfera neutra, e assim subestimativa de $d$ e conseqüente superestimativa de $z_{0}$, quando estimados pelo perfil de vento (Dolman, 1986). Padrão inverso é observado sobre instabilidade, ou seja, gradientes com inclinação inferior ao esperado em condições neutras e superestimativa de $d$ e subestimativa de $z_{0}$.

Das observações desconsideradas, três pares com valores próximos de $\zeta$ e com diferenças elevadas em $d$ e $z_{0}$ entre as observações de cada par são destacados na Figura 1. A ampli- tude de $\zeta$ para cada caso não ultrapassou 0,001 , enquanto $d$ teve diferença em módulo de até 4,08 $\mathrm{m} \mathrm{e} z_{0}$ de 1,58 m, não sendo observada relação entre os parâmetros de rugosidade e a turbulência atmosférica representada por $\zeta$. Aliado a isso, em condições de instabilidade, observou-se subestimativa de $d$ e superestimativa de $z_{0}$, ou seja, padrão inverso ao teórico. Tais resultados indicam que os elevados erros na estimava dos parâmetros de rugosidade não foram proporcionados pela influência da estabilidade na forma do perfil de vento, o que reforça o discutido anteriormente, no qual os critérios utilizados para selecionar as condições próximas à adiabática foram adequados.

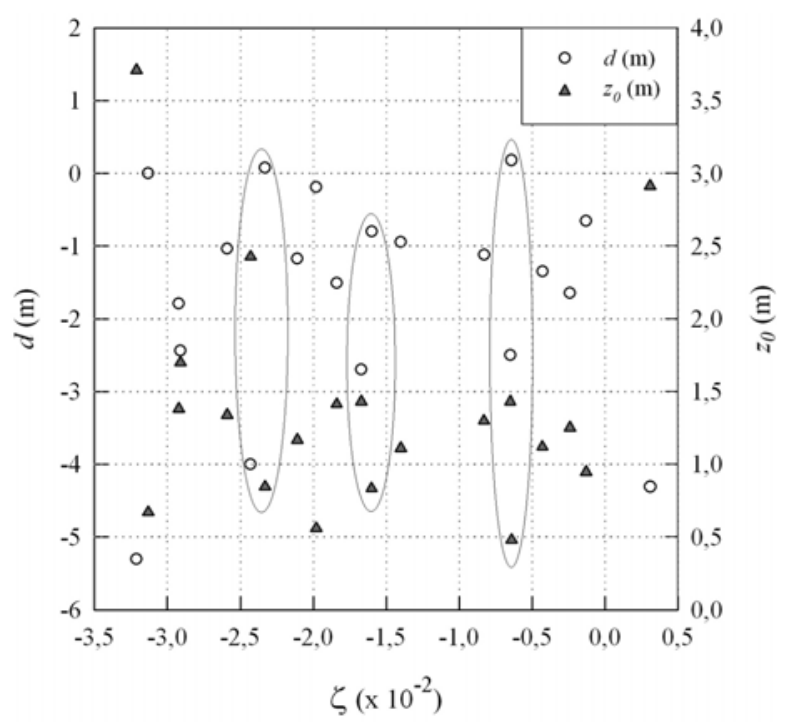

Figura 1 - Relação entre o deslocamento do plano zero $(d, \mathrm{~m})(\mathrm{o})$ e 0 comprimento de rugosidade $\left(z_{0}, \mathrm{~m}\right)(\boldsymbol{\Lambda})$ estimados pelo método convencional e a estabilidade atmosférica representada pelo parâmetro de estabilidade de MoninObukhov $\left(\zeta, \times 10^{-2}\right)$ (Monin \& Obukhov, 1954) para as observações próximas à atmosfera neutra desconsideradas.

Molion \& Moore (1983) comentam que para perfis similares e em condições de estabilidade atmosférica próximas, as estimativas de $d$ podem apresentar valores discrepantes e fisicamente inconsistentes, em função da elevada sensibilidade do método convencional a $U_{z}$. Assim como a estabilidade atmosférica, $U_{z}$ medida no anemômetro inferior $(z=3,0 \mathrm{~m})$ não teve relação com $d$ nem com $z_{0}$ para as observações desconsideradas (Fig. 2). Para essas observações, $U_{z}$ variou entre 1,1 e $4,0 \mathrm{~m} \mathrm{~s}^{-1}$, sendo que $59 \%$ foram acima de $2,0 \mathrm{~m} \mathrm{~s}^{-1}$. Valores nesse intervalo foram observados no mesmo nível de medida para as observações consideradas, com variação entre 1,7 e 3,5 $\mathrm{m} \mathrm{s}^{-1}$. 


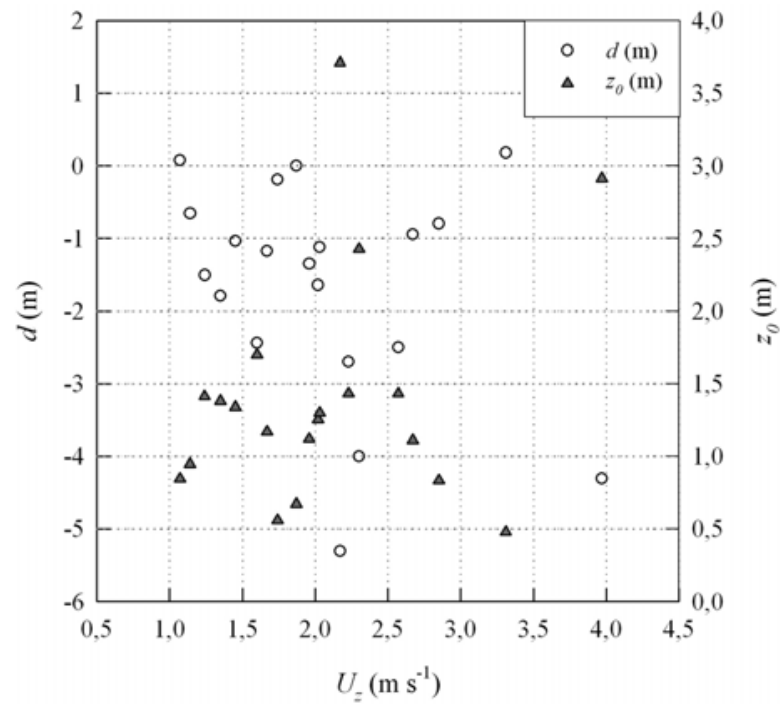

Figura 2 - Relação entre o deslocamento do plano zero $(d, \mathrm{~m})$ (o) e o comprimento de rugosidade $\left(z_{0}, \mathrm{~m}\right)$ ( $\left.\boldsymbol{\Delta}\right)$ estimados pelo método convencional e a velocidade horizontal do vento $\left(U_{z}, \mathrm{~m} \mathrm{~s}^{-1}\right)$ medida no anemômetro inferior $(z=$ $3,0 \mathrm{~m}$ ) para as observações próximas à atmosfera neutra desconsideradas.

Molion \& Moore (1983) observaram decréscimo de $d$ e $z_{0}$ com 0 aumento da velocidade do vento medida próxima ao topo do dossel, entretanto a taxa de variação de $z_{0}$ em função de $U_{z}$ foi inferior a de $d$. Este padrão é freqüentemente atribuído à flexibilidade natural da vegetação, ou ao incremento da penetração dos turbilhões e da absorção de momentum no interior da vegetação (Monteith \& Unsworth, 1990). Contudo, Molion \& Moore (1983) sugerem que a subestimativa de $U_{z}$ medida pelos anemômetros de canecas durante baixas velocidades do vento $\left(<2,0 \mathrm{~m} \mathrm{~s}^{-1}\right)$, nos níveis próximos ao dossel, resulta em superestimativa de $d$, associada à subestimativa de $z_{0}$, quando estimados pelo método convencional. Este padrão foi inverso ao apresentado para as observações desconsideradas. Assim, no presente caso, a faixa de velocidade do vento analisada não foi a fonte dos valores inconsistentes de $d$ e $z_{0}$ determinados pelo $C O$.

0 topo da subcamada rugosa pelo modelo de Wieringa (1993) foi de $3,1 \mathrm{~m}$. Valores similares foram obtidos pela expressão de Raupach (1992) (3,0 m) e pela de Jacobs \& van Boxel (1988) $(2,9 \mathrm{~m})$, ambos determinados em função dos parâmetros de rugosidade estimados no presente trabalho. Esses valores variaram em torno da altura do anemômetro inferior, ou seja, esse nível de medida estava próximo ao limite inferior de validade do perfil.

De qualquer forma, 0 intercepto $(a)$ da regressão linear entre $U_{z}$ no nível inferior de medida em função de $u_{*}\left(U_{z}=a+b u_{*}\right)$ para todas as observações com condições de atmosfera neutra foi estatisticamente não significativo $(p<0,05)$. Portanto, essa re- gressão não apresentou diferença estatística quando comparada à regressão forçada a passar pela origem $\left(U_{z}=b u_{*}\right)$ para 0 mesmo nível (Fig. 3). A não diferença estatística indicou que as medidas foram obtidas acima da SCR (Dolman et al., 1992), sendo respeitado o limite inferior de validade da lei logarítmica do vento.

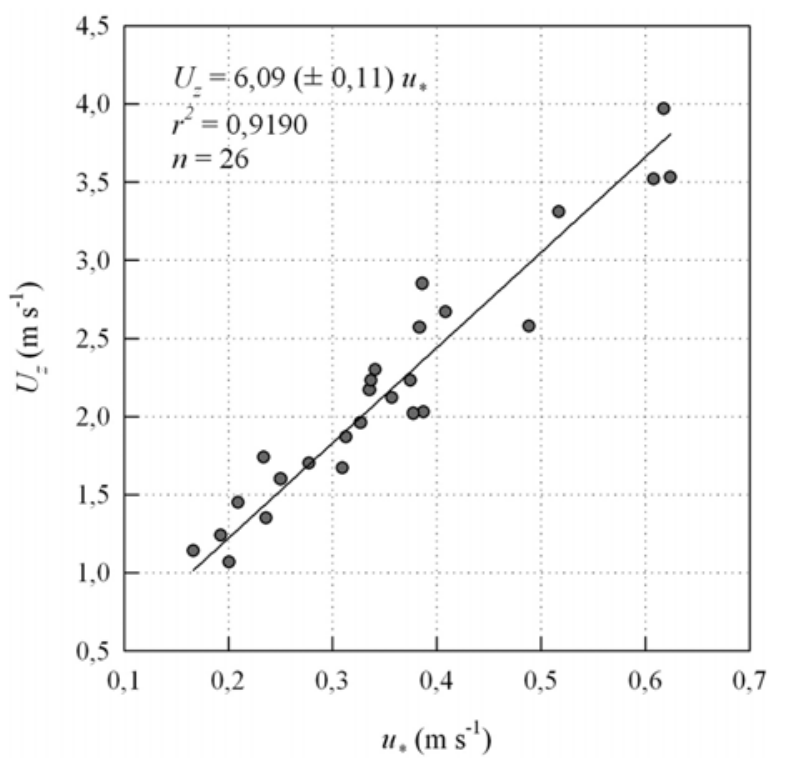

Figura 3 - Relação entre a velocidade horizontal do vento $\left(U_{z}, \mathrm{~m} \mathrm{~s}^{-1}\right)$ no nível inferior de medida $(z=3,0)$ e a velocidade de fricção $\left(u_{*}, \mathrm{~m} \mathrm{~s}^{-1}\right)$ determinada por correlação de vórtices turbulentos a 9,0 m, para todas as observações com condições de atmosfera neutra. Regressão linear $(--)$ forçada pela origem $\left(U_{z}=b u_{*}\right)$, coeficiente de determinação $\left(r^{2}\right)$ e número de amostras $(n)$. Valor entre parênteses representa 0 erro padrão de estimativa do coeficiente angular $(b)$.

A velocidade do vento apresentou direção predominante entre SE e SSE para as observações consideradas (Figs. 4A e 4B). Das desconsideradas, não houve incidência do vento entre essas direções, variando de SSE a $W$ e uma a $E$. As maiores bordaduras (> $1000 \mathrm{~m}$ ) foram entre SE e SSE, e entre WNW e NNW (Wallace et al., 1994), mas neste último intervalo de direção não ocorreu nenhum caso de atmosfera neutra. As menores bordaduras foram nos sentidos $N-S$ e $W-N E$, com aproximadamente $400 \mathrm{~m}$.

A bordadura mínima para que as medidas se localizassem no interior da subcamada inercial variou de $850 \mathrm{~m}$, para 0 anemômetro a 8,5 m acima da superfície, a $900 \mathrm{~m}$, para 0 sistema de correlação de vórtices a 9,0 $\mathrm{m}$. Esses valores atendem à relaçãa prática de 1:100 (vertical:horizontal) e ao modelo de Miyake apresentado por Wieringa (1993), com $z_{0}$ calculado no presente trabalho. Bordaduras superiores a $850 \mathrm{~m}$ não foram registradas nas direções das observações desconsideradas (Wallace et al., 

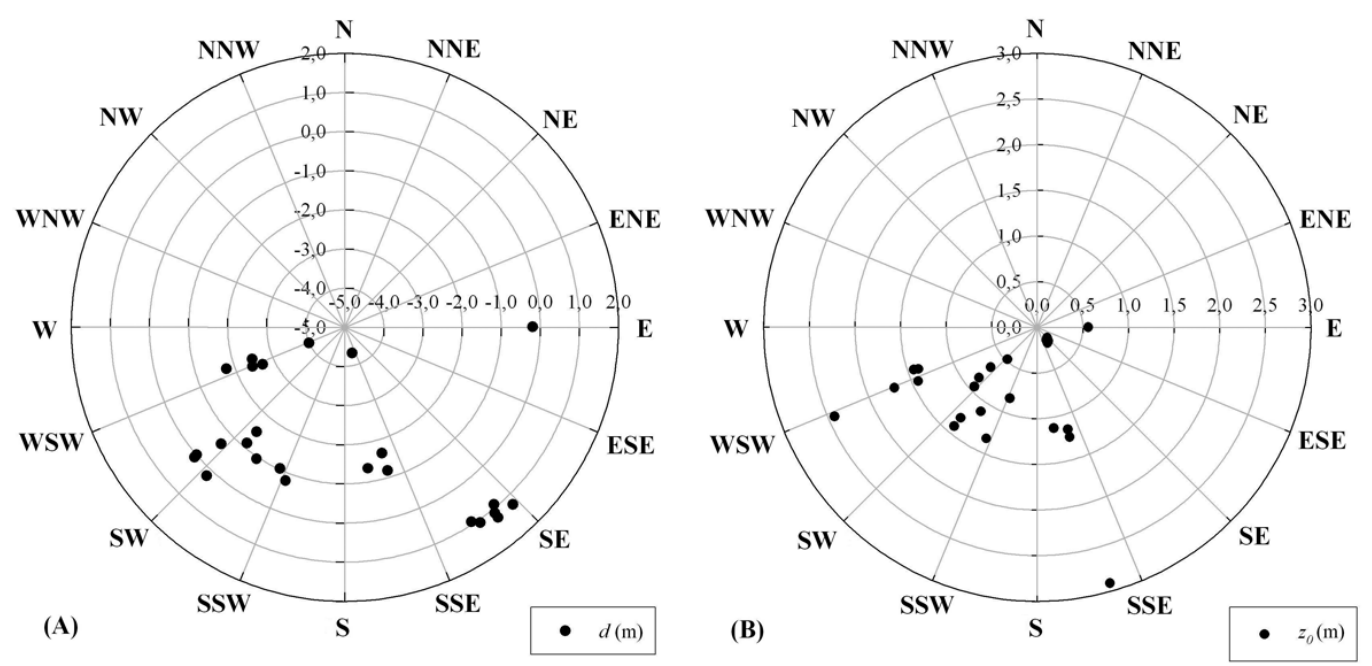

Figura 4 - Relação entre 0 comprimento de rugosidade $\left(z_{0}, m\right)(A)$ e 0 deslocamento do plano zero $(d, m)(B)$ estimados pelo método convencional e a direção do vento para todas as observações próximas à neutralidade atmostérica.

1994). Ou seja, nessas direções os fluxos são influenciados pela rugosidade regional, o que não garante que o perfil siga o predito pela teoria de similaridade de Monin-Obukhov e que representem a rugosidade da superfície subjacente (Wieringa 1993; Tsai \& Tsuang, 2005).

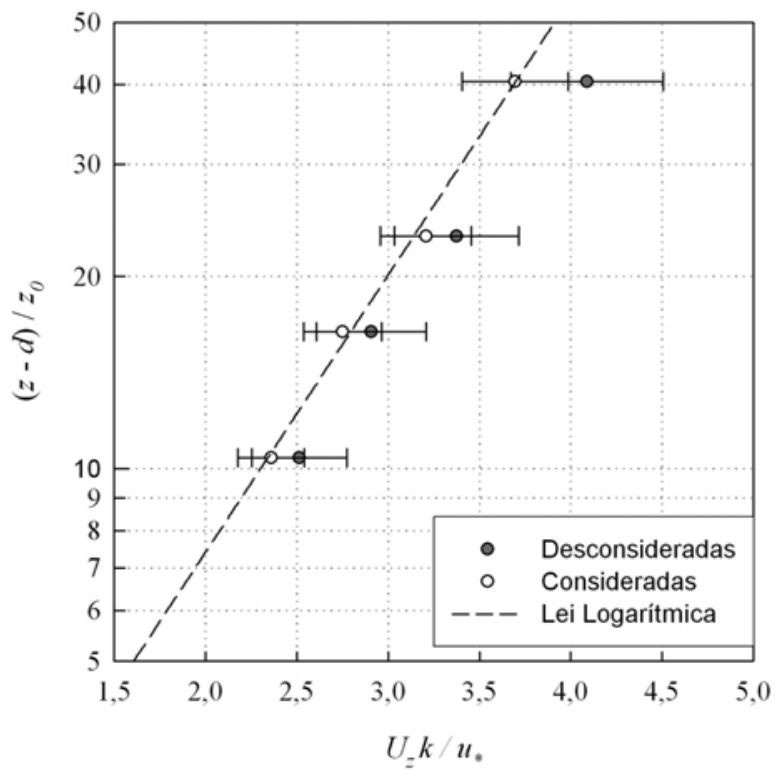

Figura 5 - Perfil de vento padronizado estimado pela lei logarítmica da velocidade do vento (--) e para os valores médios das observações com condições próximas à neutralidade atmosférica consideradas (o) e as desconsideradas (•). Barra horizontal representa o desvio padrão da média.

Quando avaliado o perfil médio do vento padronizado $\left(U_{z} k / u_{*}\right)$ em função de $d$ e $z_{0}$ determinados pelas observações consideradas (maiores bordaduras) e nas excluídas, notou-se que nos três primeiros níveis de medida $(z=3,0 ; 4,1 ; 5,3 \mathrm{~m})$ os valores de $U_{z} k / u_{*}$ para ambos os casos foram similares, com diferenças em média de 5,5\% (Fig. 5). Para esses níveis, o perfil padronizado mostrou baixa divergência do teórico estimado pela lei logarítmica, independente da direção. Porém, quando consideradas apenas as direções excluídas, a relação $U_{z} k / u_{*}$ no nível superior (8,5 m) superestimou o teórico em média 10,4\%, enquanto para as observações de maior bordadura houve concordância, com subestimativa $<1 \%$. Dessa forma, nos casos de menor bordadura, o nível superior de medida encontrou-se acima da SCI (Munro \& Oke, 1975), onde a lei logarítmica do vento não é válida, 0 que resultou nas estimativas de $d \mathrm{e} z_{0}$ inconsistentes.

As Figuras $6 \mathrm{~A}$ e $6 \mathrm{C}$ apresentam dois casos com observações próximas à atmosfera neutra e faixas similares da velocidade do vento nos três primeiros níveis de medida para as direções com bordadura insuficiente (desconsideradas) e nas de maior (consideradas). Nesses casos, as diferenças nos três primeiros níveis variaram de $-1,9$ a 2,9\%. Para as mesmas observações, $U_{z}$ medida no quarto nível e nas direções de bordadura insuficiente foi superior à observada no mesmo nível e nas direções de maior bordadura $5,2 \%$ em média. A maior velocidade do vento no anemômetro superior nas direções excluídas resultou em mudança no ajuste da curvatura do perfil de vento e maior taxa de variação média de $U_{z}$ em relação à altura (Figs. 6B e 6D). A inclinação superior de $U_{z}$ forçou a subestimativa elevada de $d$ para que a regressão entre $U_{z} \mathrm{e} \ln (z-d)$ apresentasse 0 valor máximo possível de $r^{2}$, e dessa forma, intercepto superior, o que proporcionou a superestimativa de $z_{0}$. 


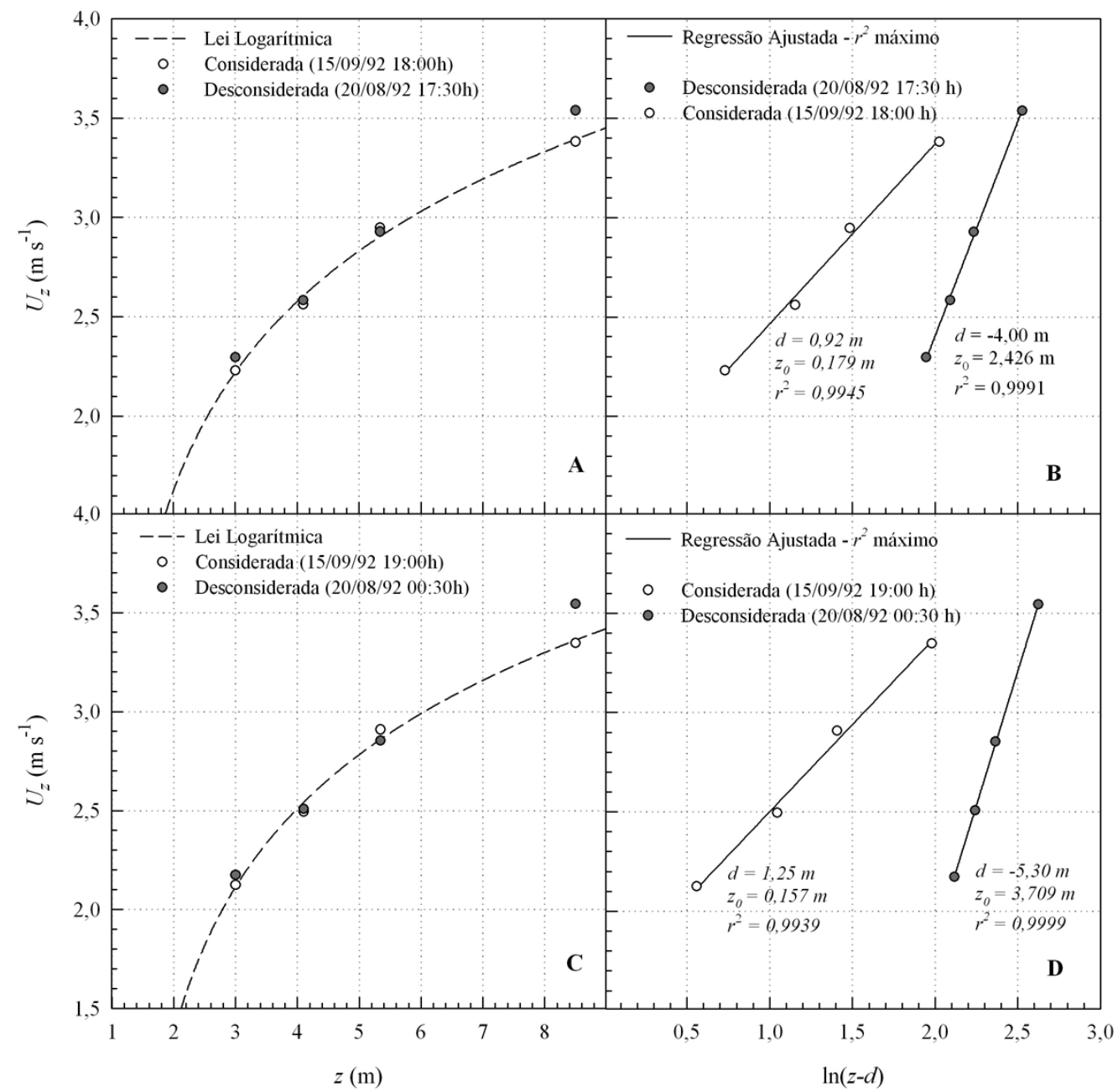

Figura 6 - Perfil vertical da velocidade do vento medido nas direções desconsideradas (•) e nas consideradas (o) e 0 estimado pela lei Iogarítmica do vento (-) utilizando os valores do deslocamento do plano zero $(d, \mathrm{~m})$ e do comprimento de rugosidade $\left(z_{0}, \mathrm{~m}\right)$ para cada observação considerada ( $\mathrm{A}$ e C). Regressão linear entre a velocidade horizontal do vento $\left(U_{z}, \mathrm{~m} \mathrm{~s}^{-1}\right)$ e $\ln (z-d)$ ajustada $(--)$ ao maior coeficiente de determinação $\left(r^{2}\right)$ possível, com $d$ e $z_{0}$ obtidos pelo método convencional, nas direções desconsideradas $(\bullet)$ e nas consideradas (o). Coeficiente de determinação da regressão $\left(r^{2}\right)$ (B e D).

0 deslocamento do plano zero padronizado pela altura dos arbustos $(d / h)$ foi de 0,53 . Este valor foi inferior ao observado para superfície esparsa, composta por arbustos e eucaliptos e uma camada de grama seca $(0,75)$, na Austrália (Garratt, 1980). Molion \& Moore (1983), utilizando um procedimento analítico baseado na conservação de massa do perfil determinaram $d / h$ $=0,65$ para as mesmas medidas de Garratt (1980). Lloyd et al. (1992) na região do Sahel, sobre cobertura vegetal similar à do presente trabalho (predominância de Guiera senegalensis L.), porém no período seco, apresentaram valor inferior $(0,40 h)$.

0 índice de área frontal das árvores $\left(\lambda_{f}=\left(2 n r_{\max } h\right) / A_{T}\right.$; área da silhueta dos obstáculos dividida pela área de solo ocu- pada pelos mesmos) para as condições de Garratt (1980) $\left(\lambda_{f}\right.$ $=0,20)$ foi similar ao observado no presente trabalho $(0,21)$, ou seja, densidade de vegetação análoga. 0 valor superior de $d / h$ apresentado por Garratt (1980) e em menor proporção pelo recalculado por Molion \& Moore (1983) podem ter sido influenciados pela distribuiçã̃o vertical da densidade foliar das árvores de eucaliptos, que mostram maior densidade próxima ao topo do dossel, contribuindo para o deslocamento de $z_{0}$ superior (Shaw \& Pereira, 1982). De qualquer forma, o valor de Garratt (1980) foi considerado elevado por Lloyd et al. (1992) para $\lambda_{f}$ observado.

0 deslocamento padronizado inferior determinado por Lloyd et al. (1992) justifica-se em parte pelas medidas efetuadas no 
período seco na região do Sahel, em que o IAF dos arbustos mostra-se inferior ao observado na estação úmida (Levy \& Jarvis, 1999). Considerando que $d$ é o nível médio de absorção de momentum (Thom, 1971), e que a diminuição da densidade de folhagem resulta em menor área de sumidouro de momentum (Dolman, 1986) espera-se diminuição de $d$.

Dolman et al. (1992) comentam que para superfície esparsa, formada por arbustos e árvores (Tiger Bush) no Sahel, o deslocamento do plano zero de 0,57 $h$ mostra-se representativo da superfície subjacente. Para vegetação semelhante à de Dolman et al. (1992), também no Sahel, de Bruin \& Verhoef (1997), utilizando as relações de similaridade para convecção livre, estimaram valor do deslocamento do plano zero de $0,53 \mathrm{~h}$. Baseado nas análises de Shaw \& Pereira (1982) e considerando a altura do máximo diâmetro do dossel $\left(h_{\max }=1,49 \pm 0,47 \mathrm{~m}\right)$ como a de sua máxima densidade, para um índice de área do dossel $(\Lambda=/ A F$ + IAG/2, área total de um único lado de todos os elementos do dossel por unidade de área superficial) medido de 0,428, obtevese $d=0,55 h$. Esses valores apresentam concordância satisfatória com o estimado no presente trabalho, sendo as diferenças inferiores a 7,6\%.

A constante de proporcionalidade $\lambda=z_{0} /(h-d)$ (Thom, 1971) foi de aproximadamente 0,189 . Para cultura artificial, $\lambda$ mostrou valor de 0,36 (Thom, 1971), sendo de 0,25 para floresta de pinus (Molion \& Moore, 1983). Para dosséis densos $(\Lambda>1$ ), Shaw \& Pereira (1982) determinaram relação linear entre $z_{0} \mathrm{e}$ $(h-d)$ e proporcional a 0,29 . Contudo, para dosséis esparsos $(\Lambda<1,0)$ essa proporção foi inferior e não constante, sendo dependente da distribuição vertical e da densidade do dossel.

Tendência análoga à observada por Garratt (1980) para $d / h$ foi mostrada por $\lambda$, com valor superior $(0,380)$ ao determinado no presente trabalho. 0 valor de $\lambda$ apresentado por Garratt (1980) foi elevado para a densidade de vegetação observada, resultado principalmente do elevado valor de $d$, visto que $z_{0} / h(0,095)$ foi próximo aos valores citados na literatura (Dolman et al., 1992; Takagi et al., 2003).

Dolman et al. (1992) utilizaram medidas do perfil de vento por radiossondagem entre 7 e $40 \mathrm{~m}$ de altura na estimativa de $z_{0}$ para $d=0,57 h$, obtendo $z_{0}=0,21(h-d)$. 0 valor de $\lambda$ em função dos resultados de Shaw \& Pereira (1982) foi de 0,21, e 0 apresentado por Molion \& Moore (1983) de 0,169. Esses valores foram próximos aos observados no presente trabalho, com diferenças em torno de 10\%. Enquanto, o determinado por Lloyd et al. (1992) foi inferior e de 0,124. 0 menor IAF para as condições de Lloyd et al. (1992) resultou em baixo valor de $d / h$, como comentado anteriormente, e de $z_{0}(0,074 h)$, que combinados, proporcionaram $\lambda$ inferior ao determinado no presente trabalho.

A média da velocidade de fricção determinada por correlação de vórtices ( $\bar{u}_{*_{-}} o b s$ ) foi de $0,449 \pm 0,150 \mathrm{~m} \mathrm{~s}^{-1}$, enquanto a média de $u_{*}$ estimada $\left(\bar{u}_{*_{-}} e s t\right)$ a cada nível de medida em função de $U_{z}$ e dos parâmetros de rugosidade foi de 0,443 \pm $0,128 \mathrm{~m} \mathrm{~s}^{-1}$. A diferença entre $\bar{u}_{*-}$ obs e $\bar{u}_{*-}$ est mostrou que de forma geral houve subestimativa em torno de $1 \%$ do valor observado. A diferença entre $\bar{u}_{*_{-}}$obs e e $\bar{u}_{*_{-}}$est foi estatisticamente não significativa pelo teste- $t(p<0,05)$. A raiz do quadrado médio do erro (RQME) para as estimativas de $u_{*}$ apresentou valor de $0,030 \mathrm{~m} \mathrm{~s}^{-1}$, que representou $6,8 \%$ da média.

A velocidade de fricção mostrou dependência diretamente proporcional a $d$ e a $z_{0}$. Porém, observou-se sensibilidade superior de $u_{*}$ a mudanças em $z_{0}$ com relação à observada para $d$. Na média do perfil, incrementos de $10 \%$ em $d$ implicaram em aumento de $u_{*}$ ao redor de $1,3 \%$, enquanto para $z_{0}$ a mesma variação resultou em diferenças de 2,9\%.

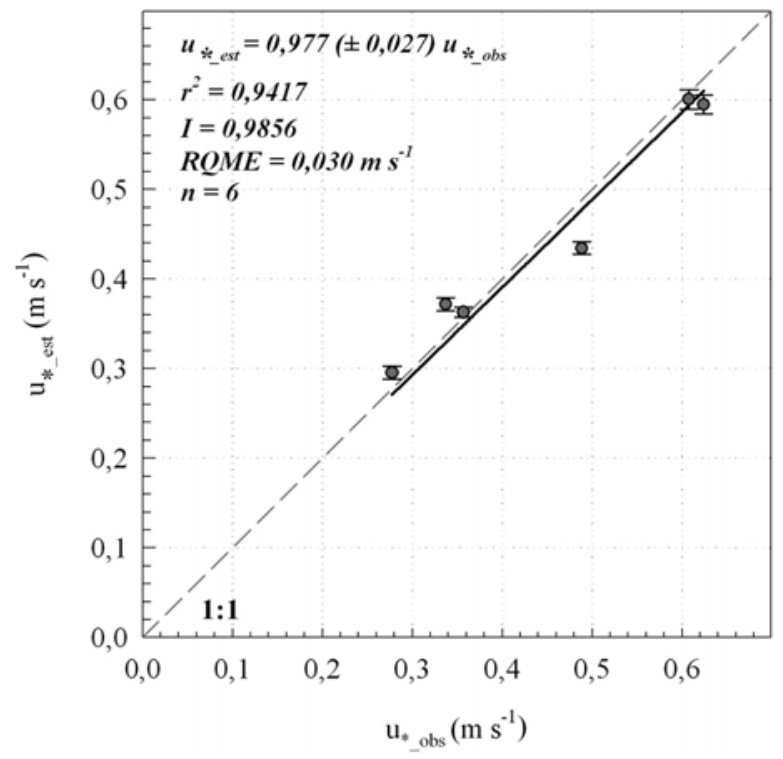

Figura 7 - Regressão linear entre a velocidade de fricção determinada por correlações de vórtices turbulentos $\left(u_{*-} o b s, \mathrm{~m} \mathrm{~s}^{-1}\right)$ e a média estimada pelo perfil logarítmico do vento $\left(u_{*-}\right.$ est, $\left.\mathrm{m} \mathrm{s}^{-1}\right)$ para cada nível de medida em função do deslocamento do plano zero e o comprimento de rugosidade determinados pelo método convencional. Valores entre parênteses representam 0 erro padrão de estimativa do coeficiente angular. Coeficiente de determinação da regressão $\left(r^{2}\right)$, índice de concordância de Willmott $(I)$, raiz do quadrado médio do erro (RQME) e número de amostras utilizadas ( $n$ ).

As regressões entre a velocidade de fricção determinada por correlação de vórtices e a estimada pela lei logarítmica mostraram interceptos não significativos estatisticamente $(p<0,05)$, assim utilizou-se nas avaliações as regressões forçadas a passar pela origem (Fig. 7). 0 valor de $r^{2}$ foi de $0,9417,0$ que 
indicou precisão satisfatória das estimativas. A velocidade de fricção estimada teve tendência de subestimar $u_{*}$ determinada por correlação de vórtices em torno de 2,3\%. Esse valor encontrouse no intervalo de incerteza ( $\sim 10 \%)$ da determinação da velocidade de fricção por correlação de vórtices turbulentos (Wyngaard, 1988). 0 índice $I$ foi de 0,9856, o que mostrou concordância satisfatória das estimativas de $u_{*}$ em relação aos valores determinados por correlaçãa de vórtices turbulentos.

\section{CONCLUSÕES}

Baseado nos resultados obtidos na determinação dos parâmetros de rugosidade aerodinâmica da superfície (deslocamento do plano zero e comprimento de rugosidade) estimados pelo método convencional sobre vegetação natural de porte alto e esparsa em ambiente semi-árido do Sahel pode-se concluir que:

- A aplicação do método é restrita pela dificuldade em se observar condições próximas à atmosfera neutra e bordadura adequada;

- Observações feitas acima da subcamada inercial resultam em parâmetros de rugosidade ou fisicamente inconsistentes ou que não caracterizam a rugosidade da superfície;

- Desde que satisfeitas as condições de validade do perfil logarítmico do vento, as estimativas do deslocamento do plano zero e do comprimento de rugosidade são satisfatórias;

- A velocidade de fricção é mais sensível a variações no comprimento de rugosidade em relação a mudanças no deslocamento do plano zero;

- 0 método convencional estima satisfatoriamente a velocidade de fricção em condições de neutralidade atmosférica.

\section{AGRADECIMENTOS}

Os autores agradecem ao Departamento de Meteorologia da Universidade de Reading, UK, pela coleta dos dados de correlação de vórtices turbulentos e de perfil de vento. Financiada pela UK Terrestrial Initiative in Global Environmental Research. Ao Instituto de Manejo Ecológico da Universidade de Edinburgh, UK, pelas análises da estrutura física da vegetação. E a equipe do sistema de informação do HAPEX-Sahel por disponibilizar os dados.

\section{REFERÊNCIAS}

BRITTER RE \& HANNA SR. 2003. Flow and dispersion in urban areas. Annual Review of Fluid Mechanics, 35: 469-496.
DE BRUIN HAR \& MOORE CJ. 1985. Zero-plane displacement and roughness length for tall vegetation, derived from a simple mass conservation hypothesis. Boundary-Layer Meteorology, 31: 39-49.

DE BRUIN HAR \& VERHOEF A. 1997. A new method to determine the zero-plane displacement. Boundary-Layer Meteorology, 82: 159-164.

DOLMAN AJ. 1986. Estimates of roughness length and zero plane displacement for a foliated and non-foliated oak canopy. Agricultural and Forest Meteorology, 36: 241-248.

DOLMAN AJ, LLOYD CR \& CULF AD. 1992. Aerodynamic roughness of an area of natural open forest in the Sahel. Annales Geophysicae, 10: 930-934.

GARRATT JR. 1980. Surface influence upon vertical profiles in the atmospheric near-surface layer. Quarterly Journal of the Royal Meteorological Society, 106: 803-819.

JACOBS AF \& van BOXEL JH. 1988. Changes of the displacement height and roughness length of maize during a growing season. Agricultural and Forest Meteorology, 42: 53-62.

KUSTAS WP, CHOUDHURY BJ, MORAN MS, REGINATO RJ, JACKSON RD, GAY LW \& WEAVER HL. 1989. Estimate of the aerodynamic roughness parameters over an incomplete canopy cover cotton. Agricultural and Forest Meteorology, 46: 91-105.

LEVY PE \& JARVIS PG. 1999. Direct and indirect measurements of LAI in millet and fallow vegetation in HAPEX-Sahel. Agricultural and Forest Meteorology, 97: 199-212.

LLOYD CR, GASH JHC \& SIVAKUMAR MVK. 1992. Derivation of the aerodynamic roughness parameters for a Sahelian savannah site using the eddy correlation technique. Boundary-Layer Meteorology, 58: 261-271.

LLOYD CR, BESSEMOULIN P, CROPLEY FD, CULF AD, DOLMAN AJ, ELBERS J, HEUSINKVELD B, MONCRIEFF JB, MONTENY B \& VERHOEF A. 1997. A comparison of surface flux at the HAPEX-Sahel fallow bush sites. Journal of Hydrology, 188/189: 400-425.

MOLION LCB \& MOORE CJ. 1983. Estimating the zero-plane displacement for tall vegetation using a mass conservation method. BoundaryLayer Meteorology, 26: 115-125.

MONIN AS \& OBUKHOV AM. 1954. Basic relationships of turbulent mixing in the surface layer of the atmosphere. Doklady Akademii Nauk SSSR, 151: 163-187.

MONTEITH JL \& UNSWORTH MH. 1990. Principles of environmental physics. (2), Edward Arnold, N.Y., 291 pp.

MORGAN DL, PRUITT WO \& LOURENCE FJ. 1971. Analyses of energy, momentum and mass transfers above vegetative surfaces. Research and Development Technical Report/ECOM, Davis, 128 pp.

MUNRO DS \& OKE TR. 1973. Estimating wind profile parameters for tall dense crops. Agricultural Meteorology, 11: 223-228. 
MUNRO DS \& OKE TR. 1975. Aerodynamic boundary-layer adjustment over a crop in neutral stability. Boundary-Layer Meteorology, 9: 53-61.

RAUPACH MR. 1992. Drag and drag partition on rough surfaces. Boundary-Layer Meteorology, 60: 375-395.

RAUPACH MR \& THOM AS. 1981. Turbulence in and above plant canopies. Annual Review of Fluid Mechanics, 13: 97-129.

RAUPACH MR, THOM AS \& EDWARDS I. 1980. A wind tunnel study of turbulent flow close to regularly arrayed rough surfaces. Boundary-Layer Meteorology, 18: 373-397.

ROBINSON SM. 1962. Computing wind profile parameters. Journal of Atmospheric Science, 19: 189-190.

SCHAUDT KJ. 1998. A new method for estimating roughness parameters and evaluating the quality of observations. Journal of Applied Meteorology, 37: 470-476.

SHAW RH \& PEREIRA AR. 1982. Aerodynamic roughness of a plant canopy: a numerical experiment. Agricultural Meteorology, 26: 51-65.

SHUTTLEWORTH WJ, GASH JHC, LLOYD CR, MCNEILL DD, MOORE CJ \& WALLACE JS. 1988. An integrated micrometeorological system for evaporation measurement. Agricultural and Forest Meteorology, 43: 295-317.

STEARNS CR. 1970. Determining surface roughness and displacement height. Boundary-Layer Meteorology, 1: 102-111.

TAKAGI K, MIYATA A, HARAZONO Y, OTA N, KOMINE M \& YOSHIMOTO M. 2003. An alternative approach to determining zero-plane displacement, and its application to a lotus paddy field. Agricultural and Forest Meteorology, 115: 173-181.

THOM AS. 1971. Momentum absorption by vegetation. Quarterly Journal of the Royal Meteorological Society, 97: 414-428.

TSAI J \& TSUANG B. 2005. Aerodynamic roughness over an urban area and over two farmlands in a populated area as determined by wind pro- files and surface energy flux measurements. Agricultural and Forest Meteorology, 132: 154-170.

VERKAIK JW \& HOLTSLAG AAM. 2007. Wind profiles, momentum flu$x e s$ and roughness lengths at Cabauw revisited. Boundary-Layer Meteorology, 122: 701-719.

WALLACE JS, BROUWER J, ALLEN SJ, BANTHORPE D, BLYTH EM, BLYTH K, BROMLEY J, BUERKERT AC, CANTWELL M, COOPER JD, CROPLEY FD, CULF AD, DOLMAN AJ, DUGDALE G, GASH JHC, GAZE SR, HARDING RJ, HARRISON RG, HOLWILL CJ, JARVIS PG, LEVY PE, LLOYD CR, MALHI YS, MASSHEDER JM, MONCRIEFF JB, PEARSON D, SETTLE JJ, SEWEL IJ, SIVAKUMAR MVK, SUDLOW JD, TAYLOR CM \& WILSON AK. 1994. HAPEX-Sahel southern super-site report: An overview of the site and the experimental programme during the intensive observation period in 1992. Institute of Hydrology, Wallingford, 55pp.

WIERINGA JA. 1980. A revaluation of the Kansas mast influence on measurements of stress and cup anemometer overspeeding. Boundary-Layer Meteorology, 18: 411-430.

WIERINGA JA. 1993. Representative roughness parameter for homogeneous terrain. Boundary-Layer Meteorology, 63: 323-363.

WILLMOTT CJ. 1981. On the validation of models. Physical Geography, 2: 184-194.

WYNGAARD JC. 1988. Flow distortion effects on scalar flux measurements in the surface layer: implications for sensor design. BoundaryLayer Meteorology, 42: 19-26.

YASUDA N. 1988. Turbulent diffusivity and diurnal variations in the atmospheric boundary layer. Boundary-Layer Meteorology, 43: 209-221.

ZHANG H, McGREGOR JL, HENDERSON-SELLERS A \& KATZFEY JJ. 2004. Impacts of land surface model complexity on a regional simulation of a tropical synoptic event. Journal of Hydrometeorology, 5: 180-198.

\section{NOTAS SOBRE OS AUTORES}

Gustavo Bastos Lyra. Graduado em Meteorologia pela Universidade Federal de Alagoas - UFAL (2000). Mestrado em Agronomia, área de concentração Meteorologia Agrícola, pela Universidade Federal de Viçosa - UFV (2002) e Doutorado em Agronomia na área de Física do Ambiente Agrícola pela Escola Superior de Agricultura "Luiz de Queiroz", Universidade de São Paulo - ESALQ/USP (2005). Atualmente é Professor Visitante do Instituto de Ciências Atmosféricas da UFAL. Atua principalmente nas áreas de processos de transporte turbulento sobre superfícies vegetadas, evapotranspiração e estatística climatológica.

Antonio Roberto Pereira. Graduado em Engenharia Agronômica pela Escola Superior de Agricultura "Luiz de Queiroz", Universidade de São Paulo - ESALQ/USP (1968). MSc e PhD em Micrometeorologia pela Purdue University, Indiana, USA (1978). Professor Titular do Departamento de Ciências Exatas, ESALQ/USP, e pesquisador em Micrometeorologia e Agrometeorologia. Bolsista do CNPq em Produtividade em Pesquisa (Nível I). 